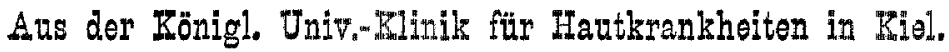 \\ (Direktor: Prof. Kling müller.)
}

\section{Über die jodophile Substanz bei dermatologischen Affektionen.}

Von

\author{
Dr. G. Stümpke,
}

Assistent der Kliniz.

Während ich in meiner letzten Arbeit ${ }^{1}$ ) über die jodophile Substanz in der Hauptsache die leukocytären Elemente bei den verschiedenartigsten Dermatosen untersuchte und mich dabei, wie es in der Natur der Sache lag, im großen und ganzen auf Ausstrichpräparate beschränken mußte, habe ich seitdem a u ch die fixen Gewebsbestandteile, also in erster Linie das Epithel und das Bindegewebe, an der Hand von Gewebsschnitten eingehender gewürdigt. Es kam mir vor allem darauf an, zu sehen, ob sich für meine Auffassung über die Bedeutung, resp. das Wesen der jodophilen Substanz, über die Bedingungen, welche ich für das Auftreten derselben als günstig und fördernd angenommen, weitere Stützen erbringen ließen. Die jodophile Metamorphose der Leukocyten konnte ich nämlich konstatieren bei hochgradigen Gewebsveränderungen, bei einer gewissen Schnelligkeit der einsetzenden Schädlichkeit und bei verhältnismäßiger Unabhängigkeit von der Blutzirkulation. Ich hatte ferner, mich stïtzend auf die Beobachtungen anderer Autoren und auf meine eigenen Untersuchungsresultate, geschlossen, daß in dem Auftreten der jodophilen Substanz, zum mindesten in der extraraskulären Jodophilie, wahr-

1) „Über Jodophilie der Leukocyten bei dermatologischen Affektionen." Berl. klin. Wochenschr. 1909. Nr. 5. 
scheinlich ein Symptomerhöhter vitaler Zelltätig keit zu erblicken sei, ohne dabei in Abrede zu stellen, dafs man für gewiße Fälle, namentlich der intravaskulären Jodophilie, z. B. bei kroupöser Pneunomie, Diabetes, auch an eine andere Interpretation denken könne. Wie denn ja überhaupt die jodophile Umwandlung der Zellen bei der Fülle der Erscheinungen, der Verschiedenartigkeit des Vorkommens nur äußerst schwer unter einen einheitlichen Gesichtspunkt zu bringen ist. An sich ist, wie ich an dieser Stelle gleich hervorbeben möchte, der Nachweis der jodophilen Substanz in Ausstrichpräparaten zweifellos leichter als in Gewebsschnitten. Einmal findet durch unsere verschiedenen Fixierungs- und Härtungsmittel eine nicht unerhebliche Schrumpfung der Gewebsstücke statt, ferner ist es möglich, daß durch Alkoholwirkung eine Modifikation in der ursprünglichen zellulären Anordnung der jodophilen Substanz vor sich geht - vgl. die diesbezügliche Mitteilung Gierkes ${ }^{1}$ ), der eine Lösung der letzteren durch wässerige Fixierungsmittel, nachfolgende Fällung durch Alkohol und Transport mittels des Diffusionsstroms an eine Zellseite annimmt. Drittens sind die Zellgrenzen an Gewebsschnitten zuweilen nicht so deutlich zu erkennen, wie an isolierten Zellen in Ausstrichpräparaten, so daß auf diese Weise die Entscheidung, ob es sich um intra- oder extrazelluläres Glykogen handelt, Schwierigkeiten begegnet. Und endlich ist natürlich die ganze Methodik weit umständlicher, als die überaus einfache und für Massenuntersuchungen ganz besonders geeignete Färbung der Ausstrichpräparate mittels der Ehrlichschen Jodgummimethode. Es liegt auf der Hand, daß die Erschwerung des exakten Glykogennachweises sich weniger bei Epithelzellen geltend machen wird, bei zellularen Elementen, die von relativer Größe, gut gegeneinander abgegrenzt, in einem geschlossenen Verband sich eingefügt finden, als vielmehr bei den dem Blute entstammenden Teilen, also in erster Linie den mono- und polynukleären Leukocyten, die in Gewebslücken eingepfercht, zwischen Zellen anderer Provenienz, zum Teil, z. B. in Infiltraten derartig dicht angeordnet, daß fast Kern an Kern zu

1) Gierke: Das Glykogen in der Morphologie des Zellstoffwechsels. Zieglers Beiträge 1904. Bd. XXXVI, p. 502. 
liegen scheint, eine genaue Sonderung bäufig unmöglich machen. Änlich liegen die Verbältnisse beim Bindegewebe, das eine exakte Rubrifizierung gleichfalls oft nicht gestattet.

Bei Berücksichtigung aller dieser Verhältnisse ist es naturgemäB schwierig, eine strikte Parallele zwischen den Befunden meiner letzten Arbeit und meinen jetzigen Untersuchungen durchzuführen. Immerhin dürfte es des Interessanten nicht ganz entbehren, verschiedene dermatologische Affektionen gewissermaßen von zwei Gesichtspunkten zu betrachten, und es wird sich trotz momentaner scheinbarer Widersprüche doch in der Mehrzahl der Fälle eine befriedigende Erklärung finden lassen.

In meinen Untersuchungen verwandte ich Gewebsstücke, welche in Alkohol, Formalin oder Sublamin fixiert, in Alkohol gebärtet und in Paraffin eingebettet waren.

Das Färbungsverfahren bestand iu der Doppeltinktion Hämatoxylin und Jodjodkaliumlösung und wurde in der Weise ausgeführt, wie es Gierke in seiner ausführlichen, oben bereits zitierten Arbeit angibt. Nachdem das Präparat die absteigende Alkoholreihe pasiert, wird es einige Minuten in eine Hämatoxylinlösung, dann in Aqua dest. gebracht, darauf 5 Minuten mit Lugolscher Liossung gefärbt, um dann nach kurzem Verweilen in $2 \%$ Jodalkohol in der üblichen Weise weiter behandelt zu werden. Gleichzeitig führten wir Kontrollfärbungen mit HämatoxylinEosin und Hämatoxylin-van Gieson aus, um bei zweifelhaften Befunden eine bessere Orientierung zu gewinnen. Der Carmin-Methode, die Gierke gleichfalls zur Färbung der jodophilen Substanz empfiehlt, welche von Best ${ }^{1}$ ) seiner Zeit angegeben wurde, haben wir uns deshalb nieht bedient, weil das Verfahren ziemlich umständlich ist, die Herstellung der Carminlösung relativ schwierig und die Haltbarkeit nur gering. Zwar werden bei Anwendung der Jodmethode auch andere Substanzen, z. B. Myelin, Lecithin gebräunt. Aber wenn man erwägt, daß auch die Carminfärbung keineswegs spezifisch wirkt, vielmehr derbes Bindegewebe, die Granula der Mastzellen gleichfalls einen roten Farbenton annehmen - auch das Zellprotoplasma der Magendrüsen und manche Kalkablagerungen sollen eine Affinität zum Carmin besitzen - so wird man wohl die Jodkaliumlösung als mindestens gleichwertig betrachten dürfen.

Ein Wort noch zur Form des in den Zellen abgelagerten Glykogens: Bekantlich hat Kaminer²) 3 Stadien der Jodreaktion unterschieden: diffuse Braunfärbung des Prop. 585.

1) Best: Über Glykogen. Zieglers Beiträge 1093. Bd. XXXIII,

2) Kaminer: Toxinämie. Deutsche med. Wochenschr. 1902. XII. 
toplasmas im Gegensatz zum weißen Kern, rotbraune Körnelung des im übrigen anders gefärbten Plasmas und endlich völlige Umwandlung des letzteren in rotbraune Körner und Schollen.

Wie ich schon in meiner früheren Arbeit darauf hingewiesen hatte, konnte ich bei der Färbung der Ausstrichpräparate mittels der Jodgummimethode die diffuse Braunfärbung des Plasmas nicht finden und ich hatte deshalb die Vermutung ausgesprochen, daß dieses erste Stadium $\mathrm{Ka}$ miners sich von dem zweiten, der rotbraunen Körnelung, nur in einer durch irgend welche äußeren Einflüsse bedingten Modifikation unterscheide. Auch jetzt, bei der Färbung von Schnittpräparaten, war ich nicht in der Lage, mich von dem Vorhandensein des sogenannten I. Stadiums zu überzeugen, konnte dagegen die Veränderungen, wie sie Kaminer als II. und III. Stadium der jodophilen Metamorphose beschrieben, bestätigen. Im übrigen ist es nach allem, was bisher über die jodophile Substanz bekannt geworden, wünschenswert, nicht an der strengen Sonderung in die einzelnen Stadien festzuhalten, sondern mehr Gewicht auf den Nachweis der eigentümlichen Zellmetamorphose an sich zu legen, als auf die Art und die Form der Plasmaveränderungen. Soweit ich die Frage zu überblicken vermag, ist es durchaus wahrscheinlich, daß speziell bei der Behandlung ron Schnittpräparaten durch die Fixierungsund Härtungsmethoden der verschiedensten Art eine Einwirkung auf das Zellprotoplasma in der Weise stattfindet, daß der ursprüngliche Modus der Glykogenverteilung dadurch wesentlich verwischt wird.

Was nun im einzelnen die von mir erhobenen Befunde anbelangt, so möchte ich zunächst die verschied enen Produkte der Lues besprechen. Bezüglich der Primäraffekte ist es mir verschiedentlich gelungen, jodophile Substanz in den atypischen Epithelwucherungen nachzuweisen, und zwar fand sie sich, soweit eine Orientierung bei den vielfach komplizierten histologischen Verhältnissen möglich, abgelagert in der Hauptsache in den oberen Schichten des Stratum Malpighi. Ferner hatte ich den Eindruck, daß die Ablagerung dort eine besonders starke war, wo der entzündliche Vorgang sich besonders

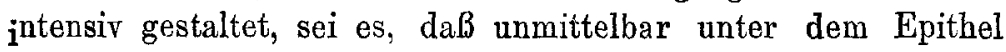


eine ausgedehnte Rundzelleninfiltration bestand, sei es, da $B$ das Epithel selbst ron leukocytären Elementen durchsetzt war. Während im ersteren Falle über den Ort der Ablagerung der jodophilen Substanz ein Zweifel nicht möglich war, indem man sie deutlich als den peripheren Teilen des Epithelzellen-Protoplasmas angehörig sah, härfig in Form der charakteristischen Kappe der Zelle förmlich aufsitzend, konnte man im letzteren Falle, besonders dann, wenn die Durchsetzung des Epithels mit Leukocyten sehr ausgeprägt war, zuweilen nicht entscheiden, in welcher von beiden Zellarten die jodophile Metamorphose sich abspielte, auch an extrazelluläre Glykogenablagerung mußte man denken. Bei dieser Gelegenheit trat mir die Schwierigkeit der exakten Lokalisation, soweit Schnittpräparate in Frage kommen, so recht klar zu Tage. Was diesen Befund der Jodophilie in syphilitischen Sklerosen anlangt, so steht er bis zu einem gewissen Grade im Gegensatz zu den Beobachtungen, welche ich in meiner vorigen Arbeit bei den Zellen des Ausstrichs-, resp. des Reizserums von Primäraffekten gemacht; es war nämlich dort nur selten der Befund der Jodophilie zu erheben. Dieser Gegensatz ist aber nur scheinbar. Denn im Reizserum sind eben andere Zellen (Leukocyten) vorhanden, während die Epithelzellen, von deren jodophiler Umwandlung wir berichteten, nur selten und in geringer Menge in jenes gelangen. Man wird nun bei billiger Würdigung der vorliegenden Verhältnisse zugeben müssen, daß man die Epithelzellen des Stratum Malpighi und die Leukocyten des Reizserums nicht ohne weiteres von einem Gesichtspunkte aus betrachten kann, daß für beide differente biologische Bedingungen vorliegen, die vielleicht für das verschiedene Verhalten bezüglich der jodophilen Substanz verantwortlich zu machen sind.

Eine ganz ähnliche Erfahrung machte ich bezüglich der Geschwüre des weichen Schankers. Während in den Leukocyten des Schankersekrets, das ich in Ausstrichpräparaten nach der Jodgummimethode färbte, sehr häufig eine ausgesprochene jodophile Umwandlung des Protoplasmas vorhanden war, habe ich in Gewebsschnitten weder in den eigentlichen Geschwürspartien noch in den Infiltraten der Umgebung eine Jodophilie zelliger Elemente nachweisen können. Wenn man 
diese Differenz nicht auf die verschiedenen Methoden selbst zurückführen will - und dafür liegt nach den sonstigen Beobachtungen keineswegs ein Anhaltspunkt vor - so wird man füglich nur die Folgerung ziehen dürfen, daß die jodophile Metamorphose nicht als irgendeinem Krankheitsbildeigentümlich, nichtals spezifischaufzufassen sei, sondern, daß man diesen Zustand der Zellen bei den allerverschiedensten Affektionen erwarten darf, wenn nur die lokalen Gewebsverhältnisse dem Auftreten der Reaktion günstig sind. Gerade der Befund bei den Ulcera mollia ist nach dieser Richtung durchaus bezeichnend, es liegt auf der Hand, daß die im Schankersekret enthaltenen Leukocyten wesentlich andere Ernährungsverhältuisse besitzen, anderen Einflüssen ausgesetzt sind, als die im Zellverbande fixierten Gewebselemente. Und es ist daher die Differenz bezüglich des Auftretens der jodophilen Substanz in keiner Weise auffallend, sondern der Ausdruck einer nach irgend einer Richtung anders gearteten Zelltätigkeit. Was die Effloreszenzen der Lues weiter anbelangt, so habe ich in den Eruptionen des Sekundärstadiums eine Jodophilie im allgemeinen nicht nachweisen können, nur bei den Condylomata lata scheint diese Zellveränderung häufig zu sein, vor allem, wenn sie relativ groß und in kurzer Zeit entstanden sind. Besonders in einem Präparate konnte ich ein geradezu prächtiges Bild einer jodophilen Metamorphose beobachten. Die Epithelzellen, alle groß und leicht ödematös, waren geradezu vollgepfropft mit dunkelbraunen Körnern, welche ziemlich regelmäßig im ganzen Protoplasma verteilt und nicht in Form einer Kappe dem Kern aufgelagert waren. Auch bei den breiten Kondylomen konnte ich die Beobachtung machen, daß der Grad der Jodophilie dort am stärksten ausgeprägt war, wo gleichzeitig eine starke entzündlicheInfiltration bestand.Immerhin muß ich hervorheben, daß die Epithelschichten, die dem Bindegewebe dicht anlagen, diese eigentümliche Umwandlung des Plasmas bedeutend geringfügiger aufwiesen. Ferner war auffallend in diesem Präparat, da B in den Epithelzellen sich wiederholt das Bild der mitotischen Kernteilung bot, daß die Kerne durchweg eine ganze Reihe von Kernkörpern enthielten und endlich, dab die Granula der Zellen des 
Stratum granulosum sich deutlich als nicht identisch mit den jodophilen Ablagerungen erwiesen. Letztere waren nämlich in den Zellen der Körnerschicht durchweg nicht vorhanden. Es ist gewiß sehr interessant, daß die gleichen hochgradigen Veränderungen, wie ich sie eben bei den breiten Kondylomen beschrieben, sich auch, und zwar fast in derselben Intensität, bei manchen Präparaten von Condylomata accuminata vorfanden. Auch hier imponierte das Freibleiben der dem Corium unmittelbar benachbarten Teile, auch hier waren die entzündlich veränderten Partien ganz besonders von der Reaktion befallen. Man wird mit Recht aus diesem analogen Verhalten bezüglich der jodophilen Metamorphose zweier ätiologisch gänzlich differenter Affektionen die oben von mir ausgesprochene Ansicht betreffend das Wesen der Jodophilie noch weiter gestützt finden.

In Eruptionen der Spätperiode der Lues habe ich die Jodreaktion vermißt. Dagegen fand ich bei einer gummösen Infiltration der Haut, die nach der Oberfäche zum Durchbruch gekommen war, zwar in dem Gummiknoten selbst keine Jodophilie und ebenso nicht in den darüber be. findlichen, größtenteils nekrotischen Epidermispartien, aber in der die Neubildung umgebenden intakten Epidermis eine kleine Strecke weit eine mäßige jodophile Metamorpbose der mittleren Epithelschichten. Eine entzündliche Infiltration dieser Epidermisteile lag nicht vor. Man wird wohl kaum fehlgehen, in diesem Ealle in der gummösen Infiltration den ätiologischen Faktor für das Auftreten der Epithelveränderung zu sehen, muß es indes wohl dahingestellt sein lassen, ob hier das Gummi schlechtweg einem Entzündungsreiz gleichzusetzen ist, oder ob einfach durch die Bildung des Infiltrats in der unmittelbaren Nachbarschaft eine Änderung der biologischen Verhältnisse des Epithels bedingt war. Eine gewisse Ähnlichkeit hat diese Beobachtung mit dem Befund Gierkes, welcher in den Randzellen einer gummösen Lebernekrose geringgradige jodophile Substanz nachwies. Alles in allem sprechen jedenfalls auch die jetzt von mir erhobenen Befunde dafür, daß bez ïglich der Jodophilie ein grundsätzlicher Unterschied zwischenden einzelnen Stadien derLuesnicht be- 
steht, sondern da $B$, wie ich auch in meiner vorigen Arbeit hervorhob, lediglich der Grad der jeweiligen Gewebsalterationen jene eigentümliche Zellveränderung bedingt.

Von weiteren Beobachtungen möchte ich noch anführen das Auftreten zahlreicher jodophiler Substanz in einer go n or rhoischen Granulationsgeschwulst des Präputium, in einem etwa bohnengroßen Angi o m des Rückens und im Epithel, das ausgedehnte Rotzgranulationen seitlich begrenzte. Im ersten Fall zeigte sich eine exquisite jodophile Metamorphose der mittleren Epithellagen des Stratum Malpighi, und zwar war dieselbe dort am deutlichsten ausgeprägt, wo die Epidermis die größte Tiefenausdehnung hatte. Bei dem MalleusPräparat beschränkte sich die Ablagerung der jodophilen Substanz auf die Epithelzellen, die den Granulationen unmittelbar benachbart waren, man hatte hier also ungefähr das gleiche Bild, wie es oben bei der gummösen Neubildung beschrieben. Das Angiom endlich charakterisierte sich dadurch, daß die Jodreaktion nur in den obersten, direkt an das Stratum granulosum grenzenden Epithelpartien nachweisbar war. Letzteres ist ron dem Gesichtspunkte aus interessant, weil die Gefäßentwicklung im Corium äußerst stark war und infolgedessen sich die Ernährungsverhältnisse für die tieferen Teile der Epidermis besonders günstig gestalteten. Wir sehen also auch hier, dab die Bildung der jodophilen Substanz in keiner direkten Abhängigkeit ron der Blutzirkulation steht. Ich verweise diesbezüglich auf die Beobachtungen Gierkes, der eine jodophile Metamorphose in normal anatomischen Gebilden gofunden, so im Knorpel und in den oberen Partien mehrschichtigen Epithels, die bei einer relativen Unabhängigkeit vom Gefäßsystem eine sozusagen selbstständigere Stoffwechseltätigkeit aufweisen.

Auch nach einer anderen Richtung ist der Befund bei dem Angiom bemerkenswert. Nachdem man wiederholt das Vorkommen ron Glykogen in Neoplasmen festgestellt, war man, da man es in einer großen Reibe von malignen Tumoren gefunden, so weit gegangen, aus dem Auftreten jodophiler Substanz Schlüsse auf die Bösartigkeit der betreffenden Geschwulst zu ziehen. Indes haben bereits Best und Gierke derartige 
Schlußfolgerungen an der Hand ihres mikroskopischen Materials als nicht zu Recht bestehend zurückgewiesen. Auch unser Fall beweist jedenfalls zur Genüge, daß Jodophilie und Malignität absolut nicht identisch zu setzen sind.

Endiich möchte ich noch erwähnen, daß ich auch bullöse Dermatosen in Gewebsschnitten untersucht habe. Wie ich seinerzeit mitgeteilt, konnte ich in Ausstrichpräparaten der verschiedenartigsten Blaseneruptionen mittels der Jodgummimethode fast regelmäßig eine deutliche Jodophilie der Leukocyten nachweisen. Die Befunde in den Serienschnitten waren nicht ganz eindeutig. Ganz abgesehen davon, daß in einem großen Teil die Methode gänzlich unzulänglich ist, sobald nämlich die Blasen eine irgendwie nennenswerte Größe besitzen, ist auch sonst in dem Detritus und geronnenen Serum des Blaseninhalts eine exakte Diagnose bezüglich der darin enthaltenen zellulären Elemente nicht eben leicht. Immerhin war es mir möglich, bei einem Ealle ron Pemphigus neonatorum eine deutliche Jodophilie der Epithelien, welche die Basis der Effloreszenz bilden, festzustellen, indes beschränkte sich die jodophilie Metamorphose auf die obersten Epithellagen und ging nicht über die Umgebung der Blase hinaus. Natürlich läßt dieser Befund nach keiner Richtung irgend welche Schlüsse zu.

Ich möchte aber doch darauf hinweisen, daß die im ganzen negativen Beobachtungen bei Blasenaffektionen sich keineswegs in Gegensatz setzen zu dem Ergebnis meiner vorigen Arbeit. Damals handelte es sich um die Leukocyten des Blaseninhalts selbst, jetzt um die fixen Gewebszellen der Blasenumgebung. Und es liegt wohl durchaus im Bereich der Möglichkeit, daß die differenten histologischen Verhältnisse, sowohl wie der Umstand, daß Zellen ganz anderer Provenienz zur Untersuchung kamen, den Unterschied in dem Auftreten der jodophilen Sub$\operatorname{stan} z$ bedingen.

Zum Schluß möchte ich noch erwähnen, daß die Befunde bei Tuberkulose durchweg negativ waren. Dabei zogen wir nicht nur die Tuberkulose der Haut heran, sondern erstreckten unsere Beobachtungen auch auf die inneren Organe, z. B. untersuchten wir Impftuberkel des Netzes, Solitärtuberkel der Cornea, 
Lungeninfiltrationen. Ich hebe diese Tatsache noch besonders hervor, weil in letzter Zeit verschiedentlich das Vorhandensein jodophiler Substanz in tuberkulösen Neubildungen beschrieben wurde. So fand Best in Riesenzellen und polynukleären Leukocyten Glykogen, auch Gierke beobachtete diese Zellmetamorphose bei Miliar- und Nierentuberkulose des Menschen und ganz besonders stark hei experimentell erzeugten Tuberkeln. Es wären also nach dieser Richtung wohl noch weitere Ergebnisse abzuwarten.

Früher hatte man allgemein angenommen, daß die Neubildungen der Tuberkulose frei von Glykogen seien und hatte eine Erklärung hierfür in dem relativen Alter des Prozesses gesucht. Indes wies schon $L$ ubarsch ${ }^{\mathbf{1}}$ ) darauf hin, daß diese Frklärung nicht so ohne weiteres Geltung haben hönne, denn er vermißte die jodophile Substanz auch in frischen Infiltraten von experimentell erzeugten Tuberkeln. Jedenfalls ersieht man auch an diesem Beispiel wieder, wie außerordentlich schwierig es ist, in allgemein giltiger Weise eine Erklärung des Wesens und der Bedeutung der jodophilen Zellmetamorphose zu liefern.

Die drei wichtigsten Vorbedingungen für die Entstehung der Jodophilie sind, wie ich bereits früher ausführte, wenigstens bei den lokalen Prozessen, e in e gew is se Unabhängigkeit ron der Blutzirkulation, eine gewisse Schnelligkeitdereinsetzenden schädigenden Ursache und drittens das Vorhandensein ziemlich hochgradiger Gewebsalterationen. Nach meinen späteren Beobachtungen möchte ich annehmen, als ob der En tz ïndung, vor allem wenn sie mehr progressiver Natur ist, sicher eine große Bedeutung beigemessen werden muß. Entzündung im Verein mit Neubildung scheint in der Tat eine gewisse Rolle zu spielen, wie meine Befunde bei Primäraffekten, Kondylomen und entzündlichen Granulationsgeschwïlsten beweisen. Dementsprechend muB ich auch, und zwar weit entschiedener als früher, die Anicht vertreten, $d$ a $B$ das Vorhandensein der jodophilen Substanzein Ausdruck erhöhter vitaler Zelltätigkeit ist.

1) Lubarsch. a) Referat. 1895. b) Über die Bedeutung der pathol. Glykogenablagerung. Virchovs Archiv, 1906. Bd. CLXXXIII. 
In Fällen von rein atrophischen Prozessen, z. B. seniler Haut, Alopecia capitis totalis, habe ich Jodophilie nicht gefunden. Rei einer Reihe ron Entzündungsvorgängen, wie ich sie in meiner vorigen Arbeit untersuchte, läßt sich aus der Art der Zellen nicht schließen, ob wir es mit einem Degenerationzustand zu tun haben oder ob ein gesteigerter vitaler Prozeß vorliegt. Dagegen sprechen die Befunde bei den entzündlichen Neubildungen, bei Tumoren wegen des häufigen Vorkommens von Kernteilungsfiguren in Zellen, die eine jodophile Metamorphose aufweisen, für eine erhöht e Zelltätigkeit.

Es unterliegt im übrigen keinem Zweifel, daß die Frage der Jodophilie wesentlich einfacher liegen würde, wenn nicht außer der extravaskulären noch eine intravaskuläre Jodophilie vorkäme. Aber gerade diese letztere, das Auftreten der jodophilen Substanz in den körperlichen Bestandteilen des Blutes und als extrazelluläres Glykogen im Serum, erschwert die Sachlage ungemein. Man kann hier keine strikten Beweise für den Charakter der zellulären Elemente finden, ist auf Theorien angewiesen und muß sogar zugeben, daß die letzten eher einer Degeneration das Wort reden. Ich rerweise diesbezüglich auf die Ausführungen in meiner vorigen Arbeit. Man wird daher vorderhand die endgiltige Entscheidung über die Bedeutung der jodophilen Zellmetamorphose noch unentschieden lassen müssen und sich dabei begnügen, das klinische resp. pathologisch-anatomische Bild immer weiter auszugestalten. Überdies möchte ich zu bedenken geben, daß die Schematisierung, auf die man bei der ganzen Frage der Jodophilie binaus will, einer streng naturwissenschaftlichen Denkungsweise bis zu einem gewissen Grade zuwiderläuft. Es gibt doch auch sonst in der Pathologie Zellveränderungen, die bei den aller verschiedensten Zuständen auftreten, eine ganz verschiedene Ätiologie besitzen und überaus schwierig zu deuten sind. So kann Fett als zellulärer Bestandteil eine Degenerationsund eine Infiltrationserscheinung sein. Die sogenannte fettige Degeneration kann auftreten als Symptom einer Entzündung, kann vorkommen bei einfachen Ernährungsstörungen und wird endlich beobachtet bei Intoxikationen der verschiedensten Art. 
Warum soll nicht ein Teil der intravaskulären Jodophilie, z. B. die Glykogenablagerungen in den Leukocyten bei Diabetes mellitus, als Resorptionsvorgang gedeutet werden, wobei die Frage, ob Degeneration, ob Zeichen erhöhter Zelltätigkeit ganz offen zu lassen wäre? Warum soll nicht bei der sogenannten diabetischen Nierenepithelquellung, dem von Ebstein seiner Zeit aufgestellten Krankheitsbild, das sich durch eine Glykogenablagerung in den Epithelien der Henleschen Schleifen charakterisiert, eine Resorption zuckerhaltigen Urins in Frage kommen?

Ich glaube, man tut der ganzen Frage Gewalt an, wenn man z. B. in diesen beiden Fällen entweder nur herabgesetzte oder nur gesteigerte Zelltätigkeit annimmt. Ganz eng hängt hiermit natürlich auch die Frage nach der Herkunft der jodophilen Substanz zusammen. Gewiß läßt sich nicht leugnen, daß speziell bei Befunden, wie ich sie eben erwähnt, die Annahme einer Zufuhr von außen ihre Berechtigung hat. Indes wird doch die überwiegende Mehrzahl von Beobachtungen, vor allem bei der extravaskulären Jodophilie, fü $\mathbf{r}$ e in e $\mathbf{n}$ selbstständigen Vorgang in der Zelle sprechen, so daß dann also die jodophile Metamorphose als das Endstadium eines Stoffwechselvorgangs zu betrachten wäre. Irgendwelche differentialdiagnotische Schlüsse aus dem Auftreten der Glykogenablagerungen zu ziehen, halte ich für nicht angängig. Man kann wohl sagen, der Befund jodophiler Substanz spricht gegen einen rein atrophischen Prozeß, man kann aber nicht umgekehrt aus dem Vorkommen jodophiler Zellmetamorphose ein gewisses Fortschreiten der betreffenden Gewebsveränderung folgern, und nun gar auf der Basis dieses zellulären Vorgangs eine Abgrenzung einzelner Aftektionen gegeneinander durchzuführen, erscheint mir zum mindesten ein etwas problematischer Versuch. Es sei bemerkt, da $B$ auch von anderer Seite, z. B. von Küttne ${ }^{1}$ ) derartige Bestrebungen, die Jodreaktion differentialdiagnotisch zu verwerten, mit großer Skepsis aufgenommen werden.

1) Küttner. Über die Jodreaktion der Leukocyten und ihre chirurgische Bedeutung. Archiv für klinische Chirurgie. 1904. Bd. LXXIII. 